\title{
Increased Adiposity Does Not Exacerbate Impaired Vasodilation in Rats Exposed to Eucapnic Intermittent Hypoxia
}

\author{
Karen L. Sweazea Nancy L. Kanagy Benjimen R. Walker \\ Vascular Physiology Group, Department of Cell Biology and Physiology, University of New Mexico Health Sciences \\ Center, Albuquerque, N. Mex., USA
}

\section{Key Words}

Adiposity · Eucapnic intermittent hypoxia $\cdot$ High-fat diet $\cdot$ Hypoxia $\cdot$ Oxidative stress $\cdot$ Sleep apnea $\cdot$ Vasodilation

\begin{abstract}
Background: Although there often is a clinical co-incidence of increased adiposity and obstructive sleep apnea, each factor is independently associated with elevated oxidative stress. Objective: We hypothesized that overweight rats exposed to simulated sleep apnea would develop exacerbated oxidative stress leading to impaired endothelium-dependent vasodilation. Methods: Rats were fed either a chow or high-fat diet (HFD; $60 \%$ kcal from fat) for 6 weeks. During the final 14 days of each diet, animals were exposed to either air or eucapnic intermittent hypoxia (E-IH) to simulate sleep apnea. Results: Rats exposed to either E-IH or HFD alone showed increases of 161 and $176 \%$, respectively, in oxidative stress (measured as thiobarbituric acid-reactive substances) compared to chow + air controls. However, oxidative stress was lower following combined HFD + E-IH treatment $(132 \%$ of chow + air controls) compared to each individual treatment. All three treatment groups, chow $+\mathrm{E}-\mathrm{IH}, \mathrm{HFD}+\mathrm{air}$ and $\mathrm{HFD}+\mathrm{E}-\mathrm{IH}$, had increased blood pressure $(144.5 \pm 4.4,148.2$ \pm 5.6 , and $136.2 \pm 2.0 \mathrm{~mm} \mathrm{Hg}$, respectively, vs. chow + air:
\end{abstract}

$123 \pm 2.0 \mathrm{~mm} \mathrm{Hg}$ ) and attenuated acetylcholine (ACh)-mediated vasodilation $(78.3,72.7$, and $78.2 \%$ of the chow + air response at the highest dose of $\mathrm{ACh}$ ) compared to chow + air controls. Combined HFD and E-IH treatment did not further impair vasodilation compared to chow $+\mathrm{E}-\mathrm{IH}$ alone. Vasodilatory responses were normalized by the antioxidant EUK134 in each treatment group. Conclusions: Increased adiposity and simulated sleep apnea impair endotheliumdependent vasodilation through enhanced generation of reactive oxygen species (ROS). However, the combined treatment does not exacerbate either ROS generation or vascular dysfunction observed with HFD or E-IH alone.

Copyright $\odot 2010$ S. Karger AG, Basel

\section{Introduction}

Reactive oxygen species (ROS) contribute to many cardiovascular diseases by causing impaired endotheliumdependent vasodilation, atherosclerosis, cardiac hypertrophy and cardiomyocyte apoptosis [1-6]. Superoxide $\left(\mathrm{O}_{2}^{\overline{2}}\right)$ impairs endothelium-dependent vasodilation by scavenging nitric oxide (NO) to form peroxynitrite $\left(\mathrm{ONOO}^{-}\right)$[6]. It may also oxidize the NO synthase (NOS) co-factor, tetrahydrobiopterin, leading to uncoupling of

\section{KARGER}

Fax +41613061234 E-Mail karger@karger.ch www.karger.com
(C) 2010 S. Karger AG, Base

0025-7931/11/0811-0047\$38.00/0

Accessible online at:

www.karger.com/res
Karen Sweazea, $\mathrm{PhD}$

Healthy Lifestyles Research Cente

College of Nursing and Health Innovation, Arizona State University

401 E Tyler Mall, MSC 4501, Tempe, AZ 85287 (USA)

Tel. +1 480965 6025, Fax +1 480968 4399, E-Mail Karen.Sweazea@ asu.edu 
the enzyme [reviewed in ref. 7]. Through this mechanism, $\mathrm{O}_{2}^{-}$decreases the bioavailability of NO $[8,9]$ thereby impairing vasodilation. Another ROS with vasoactive properties is $\mathrm{H}_{2} \mathrm{O}_{2}$, which is a potent oxidant with reported vasodilatory or vasoconstrictor effects depending on the concentration or tissue examined [10-12]. Due to these effects of ROS, a balance between the production of ROS and their scavenging by endogenous antioxidant pathways is integral to maintenance of normal vascular function [12].

Sleep apnea is characterized by repeated episodes of breathing cessation and is linked with elevated oxidative stress $[1,13]$ and increased cardiovascular morbidity [1]. The most common form of this disorder affecting 2-4\% of the population is obstructive sleep apnea (OSA), which is often associated with obesity but is also seen in elderly individuals with normal body mass indices [14-17]. Patients with OSA not only develop increased ROS levels but also impaired glucose tolerance, which can lead to insulin resistance [14, 18-20]. These effects of OSA on glucose tolerance are likewise observed in rodent models, where lean, high-cholesterol diet-fed or genetically obese mice exposed to intermittent hypoxia (IH) develop impaired glucose tolerance $[21,22]$. Similarly, IH increases oxidative stress in both lean mice and mice fed a highcholesterol diet for 84 days. In these latter studies, oxidative stress appeared to be a consequence of IH rather than diet, since it was not observed in animals fed high cholesterol alone [22].

Elevated production of ROS in OSA patients likely contributes to vascular dysfunction [23]. For example, OSA patients exhibit impaired endothelium-dependent vasodilation (measured by reduced forearm blood flow) compared to obese control patients [24]. Similarly, endothelium-dependent flow-mediated vasodilation is impaired in patients with OSA compared to control subjects $[25,26]$. Normalization of vasodilatory responses by intravenous injection of the antioxidant vitamin $\mathrm{C}$ suggests impaired vasodilation is due to elevated oxidative stress [27].

Similar to OSA, obesity is clearly linked to oxidative stress and impaired vasodilation $[28,29]$. For example, rabbits fed a high-cholesterol diet exhibit a strong negative correlation between ROS and endothelium-dependent vasodilation [30]. Similarly, rats fed a high-fat diet (HFD; 60\% kcal from fat) for 6 weeks demonstrate elevated oxidative stress and impaired endothelium-dependent vasodilation that is normalized by antioxidants [31]. However, the potential interaction between IH exposure and increased adiposity has not been examined. The goal of the present study, therefore, was to test the hypothesis that combined increased adiposity and $\mathrm{IH}$ leads to more profound oxidative stress and impaired vasodilatory reactivity than observed with either condition alone.

\section{Experimental Procedures}

\section{Study Design}

All protocols were reviewed and approved by the Institutional Animal Care and Use Committee of the University of New Mexico Health Sciences Center. Male Sprague-Dawley rats (140-160 g body weight; Harlan Industries, Indianapolis, Ind., USA) were divided into two groups and fed either a chow diet (diet 2018; 3.4 total kcal/g; Harlan Teklad Global Diets, Madison, Wisc., USA) containing $57.33 \%$ carbohydrates, $18.9 \%$ protein and $5 \% \mathrm{kcal}$ fat, or HFD (diet D12492; 5.24 total kcal/g; Research Diets, New Brunswick, N.J., USA) consisting of $20 \%$ carbohydrates, $20 \%$ protein, and $60 \% \mathrm{kcal}$ fat (mainly lard). Rats were maintained on the respective diets for 6 weeks. During the final 14 days of each diet, rats were exposed to either air or eucapnic IH (E-IH) as described previously $[32,33]$. Briefly, animals were housed in Plexiglas cages with free access to food and water. To simulate the E-IH conditions of sleep apnea, rats were exposed to repeated episodes of a 90 -second stream of $\mathrm{N}_{2}-\mathrm{CO}_{2}$ to reach an ambient nadir of $5 \% \mathrm{O}_{2}: 5 \% \mathrm{CO}_{2}$ followed by a 90 -second air flush for $7 \mathrm{~h} /$ day. Control rats were exposed to air-air cycling for $7 \mathrm{~h} /$ day. The nadir of $5 \% \mathrm{O}_{2}$ was chosen to simulate systemic hypoxemia observed in OSA patients in whom minimum $\mathrm{O}_{2}$ saturations of $\sim 70 \%$ are seen $[34,35]$. The $\mathrm{CO}_{2}$ level used has been shown previously in our laboratory to maintain eucapnia [36]. Animals were housed in identical cages in the same animal facility and exposed to a 12:12 $\mathrm{h}$ light-dark cycle. Rats were weighed prior to and following each treatment. At the end of the treatment protocols, epididymal fat pad mass was measured to gauge adiposity and systolic blood pressure was assessed by tail cuff plethysmography. Plasma samples were collected by cardiocentesis following the 14-day exposure to either air or E-IH. Samples were collected during the morning in rats deeply anesthetized with sodium pentobarbital prior to removal of mesenteric arteries for vascular studies as described below. Radioimmunoassay measurements of plasma corticosterone (the major glucocorticoid in rodents [37]) and leptin were performed by the Hormone Assay and Analytical Services Core Facility at Vanderbilt University (Nashville, Tenn., USA). Plasma nitrates and nitrites (NOx) were measured using a commercially available kit (780001; Cayman Chemical, Ann Arbor, Mich., USA) according to the manufacturer's protocol. Plasma thiobarbituric acid-reactive substances (TBARS), an indirect marker of lipid peroxidation, were analyzed using a kit from OXItek according to the manufacturer's protocol (catalogue No. 0801192; ZeptoMetrix, Buffalo, N.Y., USA).

\section{Methods}

Preparation of Experimental Solutions. The cell-permeant ROS-sensitive fluorescent indicator, 5-(and-6)-chloromethyl$2^{\prime}, 7^{\prime}$-dichlorodihydrofluorescein diacetate, acetyl ester (DCF; Molecular Probes, Carlsbad, Calif., USA), was dissolved in anhydrous dimethyl sulfoxide (DMSO) at a concentration of $50 \mu \mathrm{g} / \mathrm{ml}$. Immediately prior to loading, DCF was mixed with a $20 \% \mathrm{v} / \mathrm{v}$ so- 
lution of pluronic acid in DMSO, and this mixture was diluted with HEPES buffer to yield a final concentration of $5 \mu \mathrm{M} \mathrm{DCF}$ and $0.05 \%$ pluronic acid. Phenylephrine (PE) and acetylcholine (ACh; Sigma-Aldrich, St. Louis, Mo., USA) were dissolved in deionized water, aliquoted, and frozen $\left(-20^{\circ} \mathrm{C}\right)$ until use.

Oral Glucose Tolerance Tests (OGTT). OGTT were performed 1 day prior to euthanasia and collection of isolated arteries (below). Rats were food restricted by providing $4 \mathrm{~g} / \mathrm{rat}$ of their respective diets at 5 p.m. the night prior to the OGTT. After an initial tail vein blood sample was drawn at 8 a.m. the following morning ( $0 \mathrm{~min})$, rats were administered $1 \mathrm{~g} / \mathrm{kg} \mathrm{D}$-glucose by gavage. Blood samples were taken 0,60 , and $120 \mathrm{~min}$ after glucose administration for analysis of whole-blood glucose using a spectrophotometric glucose meter (CardioChek PA; Polymer Technology Systems, Indianapolis, Ind., USA).

Isolation of Mesenteric Resistance Arteries. Rats were anesthetized with sodium pentobarbital $(200 \mathrm{mg} / \mathrm{kg}$, i.p.) and a midline laparotomy performed to remove the mesenteric arcade. Mesenteric arteries were chosen since they are known to contribute significantly to overall blood pressure regulation [38] and are representative of systemic vascular reactivity. The arcade was immediately placed in ice-cold HEPES buffer (in mM: $134.4 \mathrm{NaCl}, 6 \mathrm{KCl}$, $1 \mathrm{MgCl}_{2}, 1.8 \mathrm{CaCl}_{2}, 10$ HEPES, 10 glucose, pH 7.4 with $\mathrm{NaOH}$ ) pinned out in a Silastic-coated dissection dish, and fifth-order mesenteric resistance arterioles ( $\sim 1 \mathrm{~mm}$ length; $80-120 \mu \mathrm{m}, \mathrm{i} . \mathrm{d}$. were dissected free. Isolated arterioles were transferred to a HEPES-filled vessel chamber (CH-1; Living Systems Instrumentation, Burlington, Vt., USA), cannulated with glass pipettes, and secured in place with silk ligatures. The vessels were stretched longitudinally to approximate in situ length, pressurized to 60 $\mathrm{mm} \mathrm{Hg}$ with either a buffer-filled column or servo-controlled peristaltic pump (Living Systems Instrumentation), and the chamber placed on a microscope stage. Vessels were superfused during each experimental protocol with warm physiological saline solution (PSS) at $37^{\circ} \mathrm{C}$ containing (in mM): $129.8 \mathrm{NaCl}, 5.4$ $\mathrm{KCl}, 0.5 \mathrm{NaH}_{2} \mathrm{PO}_{4}, 0.83 \mathrm{MgSO}_{4}, 19 \mathrm{NaHCO}_{3}, 1.8 \mathrm{CaCl}_{2}$, and 5.5 glucose at a rate of $10 \mathrm{ml} / \mathrm{min}$. The PSS was aerated with $21 \% \mathrm{O}_{2}$, $6 \% \mathrm{CO}_{2}$, balance $\mathrm{N}_{2}$ gas mixture throughout the experiments to maintain oxygenation and $\mathrm{pH}$. Therefore, all studies were conducted under standardized normoxic conditions. Arterial viability was verified prior to each experiment by noting the responses to the vasoconstrictor $\mathrm{PE}\left(10^{-6} \mathrm{M}\right)$ and to the endothelium-dependent vasodilator $\mathrm{ACh}\left(10^{-6} \mathrm{M}\right)$.

Measurement of Vascular ROS. Vessel chambers were placed on a Nikon Diaphot 300 microscope and examined with a $10 \times$ fluorescence (FITC) objective. Following $30 \mathrm{~min}$ of equilibration in aerated PSS, vessels were loaded at $37^{\circ} \mathrm{C}$ with DCF in the dark in a temperature-controlled vessel chamber (Living Systems Instruments). DCF is oxidized by cytoplasmic peroxynitrite $\left(\mathrm{ONOO}^{-}\right)$, hydrogen peroxide $\left(\mathrm{H}_{2} \mathrm{O}_{2}\right)$, and hydroxyl radicals $(\cdot \mathrm{OH})$ to produce a fluorescent product [39-41]. Images were collected prior to DCF loading (for background) and $50 \mathrm{~min}$ later using a cooled, digital CCD camera (SenSys 1400; Photometrics, Tucson, Ariz., USA). Images were processed with MetaFluor 4.5 software (Molecular Devices, Sunnyvale, Calif., USA). Sensitivity of DCF fluorescence as a measure of ROS was verified in prior experiments by noting the inhibitory effect on the signal resulting from a combination of $10 \mathrm{~mm}$ tiron (a superoxide dismutase mimetic) and 1,200 U/ml catalase [5]. Previous studies from our laboratory have also demonstrated increased DCF fluorescence in isolated arterioles in response to xanthine/xanthine oxidase which augments ROS production [42]. Since DCF may leak from cells, fluorescence from the extravascular solution was subtracted from the total to estimate intravascular ROS [40].

ACh-Induced Vasodilation. In separate experiments, vessel chambers were transferred to an inverted microscope (model TMS; Nikon) equipped with a $10 \times$ objective, video camera and monitor for analysis. Arteries remained pressurized at $60 \mathrm{~mm} \mathrm{Hg}$ for a 30-min equilibration period and were then superfused for $1 \mathrm{~h}$ with either control PSS or PSS containing chloro[[2,2'-[1,2ethanediylbis[(nitrilo-KN)methylidyne] $b i s[6-m e t h o x y p h e n o l a-$ to-KO]]]-manganese (EUK-134, $10 \mu \mathrm{M}$; Cayman Chemical). EUK-134 is a synthetic manganese-porphyrin complex with antioxidant properties as a superoxide dismutase and catalase mimetic to scavenge ROS [43]. It is also an effective scavenger of lipid peroxides [44]. Vessels were then constricted to $50 \%$ of resting inner diameter with PE. Subsequent vasodilation in response to increasing concentrations of ACh $\left(10^{-9}\right.$ to $10^{-5} \mathrm{M}, 3$ min each step) was then assessed by measuring intraluminal diameter using a video dimension analyzer (Living Systems). Data were digitized using DATAQ A/D software for analysis (DATAQ Instruments, Akron, Ohio, USA).

Analysis. Data are expressed as means \pm SEM. Potential differences in DCF fluorescence were examined by one-way analysis of variance (ANOVA). When significance was indicated, groups were compared using Tukey post hoc analysis. Percent vasodilation to ACh was calculated as the percent difference of intraluminal diameter observed at each concentration versus calcium-free PSS. Prior to analysis, percentage data were arcsine transformed to approximate a normal distribution. Data from vasodilation experiments were analyzed by two-way repeated-measures ANOVA. Where significant differences were indicated, individual groups were compared using Student-Newman-Keuls post hoc analysis. A probability value $\leq 0.05$ was accepted as statistically significant for all comparisons.

\section{Results}

\section{Effects of Treatment on Body Mass, Adiposity, and \\ Plasma Hormone Levels}

There were no significant differences in body mass between rats prior to beginning each feeding protocol (data not shown) or between rats in similar feeding protocols prior to exposure to air-air and E-IH cycling (fig. 1a). By the end of the 6-week protocol, rats fed HFD and exposed to air-air cycling gained significantly more weight than chow + air rats. Exposure to E-IH diminished weight gain in rats fed either diet (fig. 1a). There was no significant difference in body mass between chow and HFD rats exposed to E-IH (fig. 1a). Overall, HFD rats developed significantly more adipose tissue compared to the chow rats regardless of treatment (fig. 1b). Adiposity was measured using the epididymal fat pad as it can be easily extracted objectively and reflects overall increases in adiposity. Rats exposed to E-IH not only gained less weight than the 


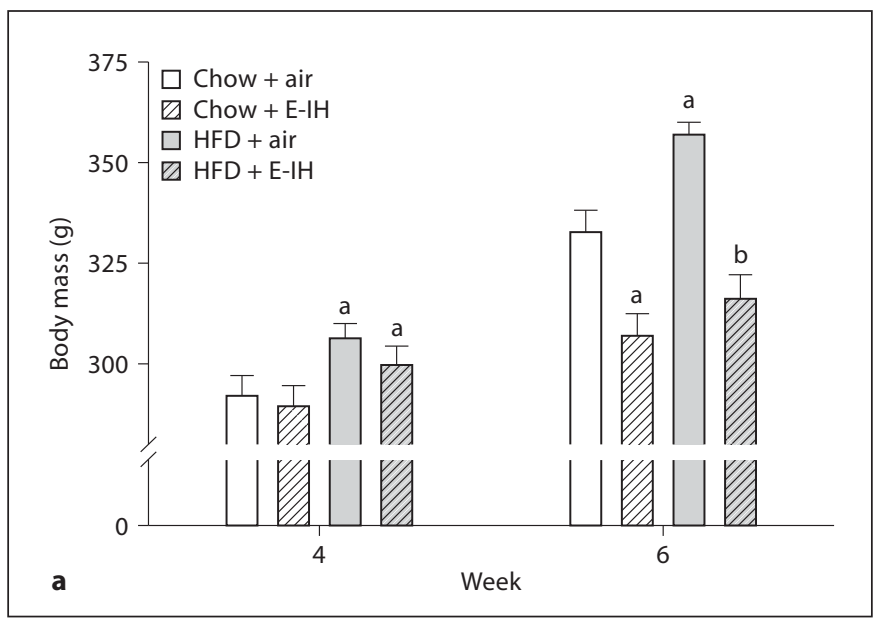

Fig. 1. a Body mass measurements during the 6-week study protocol. Body mass of rats measured before starting respective diets $(0$ week) was not different between groups (data not shown) and was increased in all groups at week 4, prior to air or E-IH exposure. At week 6, after 14 days of E-IH exposure, only air rats had a further

air-exposed animals, but also demonstrated less adiposity (fig. 1b).

Exposure to E-IH did not affect plasma corticosterone levels in chow-fed rats but levels were significantly increased in HFD-fed rats compared to chow + air and chow + E-IH animals. No significant difference in plasma corticosterone levels was observed between HFD + E-IH and HFD + air rats (fig. 2a). High-fat feeding resulted in significantly greater plasma leptin compared to chow + air or E-IH rats. Despite the diminished weight gain, plasma leptin levels were not significantly decreased in rats treated with the HFD + E-IH combination compared to HFD treatment alone (fig. 2b).

Oral Glucose Tolerance Test. HFD + E-IH rats had significantly greater initial fasting (time 0 ) blood glucose compared to HFD + air, chow + air and chow + E-IH rats (fig. 3). Blood glucose levels in HFD + E-IH rats were not affected by the oral glucose load, whereas blood glucose levels in chow + air, chow + E-IH and HFD + air rats demonstrated a significant increase at $60 \mathrm{~min}$ but returned to fasting levels at the conclusion of the OGTT (fig. 3).

\section{Measurement of ROS}

Plasma TBARS was greater in both HFD and E-IH treatments compared to chow control (fig. 4a). Plasma TBARS were also elevated with the combined stimulus of HFD + E-IH compared to the chow + air group; however, TBARS were lower in this combined group compared to

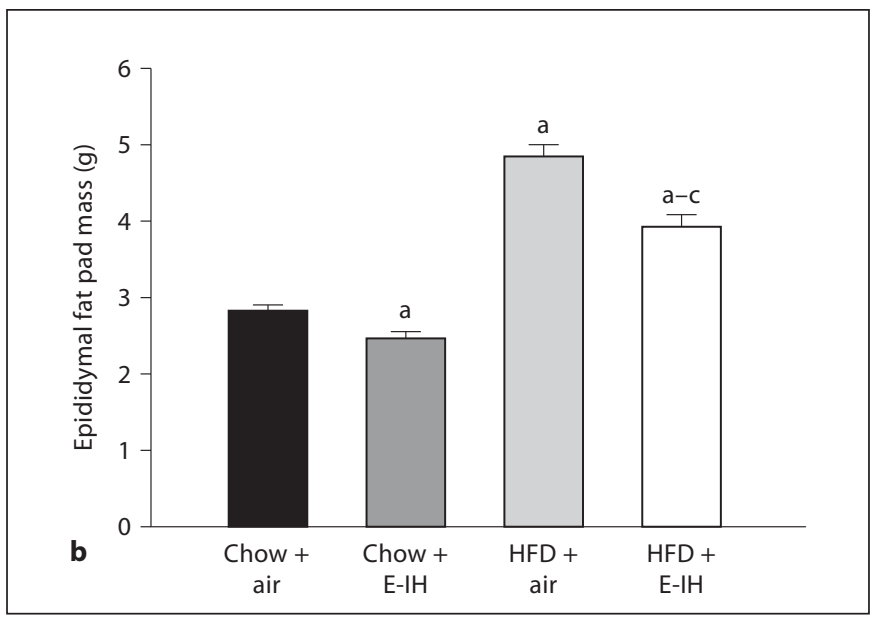

increase in body mass. $\mathbf{b}$ Epididymal fat pad mass following air or E-IH treatment in chow and HFD groups. Diminished weight gain in E-IH rats was accompanied by decreased adiposity in each feeding protocol. Means \pm SEM. $n=14-23$; $^{\mathrm{a}} \mathrm{p}<0.05$ vs. chow + air; ${ }^{\mathrm{b}} \mathrm{p}<0.05$ vs. HFD + air; ${ }^{\mathrm{c}} \mathrm{p}<0.05$ vs. chow $+\mathrm{E}-\mathrm{IH}$.
HFD alone. A similar pattern existed in DCF fluorescence in isolated arterioles (fig. 4b). Again, vessel ROS were greater in both the HFD and E-IH groups compared to chow + air controls. However, ROS were not different from chow + air controls in the combined stimulus (HFD + E-IH) group (fig. 4b).

\section{Blood Pressure and Plasma NOx}

Systolic blood pressure was greater in all treatment groups (chow + E-IH, HFD + air, and HFD + E-IH) compared to chow + air controls (fig. 5a). In contrast, circulating NOx levels were significantly higher in HFD + E-IH rats compared to both chow + E-IH and HFD + air rats (fig. 5b). Plasma levels of NOx were not significantly different in chow + air, chow + E-IH or HFD + air rats (fig. 5b).

\section{ACh-Induced Vasodilation}

Effect of EUK-134 in Arteries from Chow + Air Controls. ACh caused concentration-dependent dilation in vessels preconstricted to $50 \%$ of resting inner diameter with PE. This vasodilatory response to ACh was modestly inhibited by treatment with the ROS scavenger EUK-134 (fig. 6a). These data suggest that ACh-induced vasodilation may be mediated, in part, through one or more ROS species in vessels from chow + air control rats.

Effect of EUK-134 in Arteries from Chow + E-IH Rats. Vasodilatory responses to ACh were impaired in small 

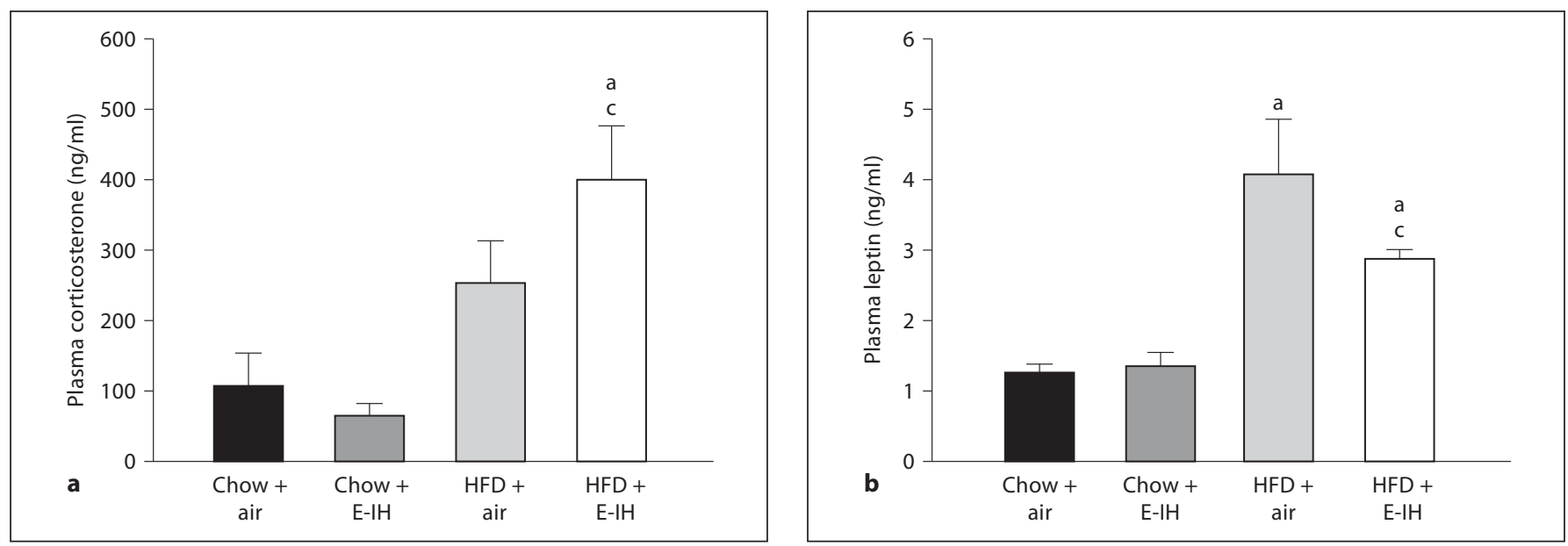

Fig. 2. Plasma corticosterone (a) and plasma leptin (b) levels from rats following each treatment protocol. Means \pm SEM. ${ }^{\mathrm{a}} \mathrm{p}<0.05$ vs. chow $+\mathrm{air} ;{ }^{\mathrm{b}} \mathrm{p}<0.05$ vs. HFD + air; ${ }^{\mathrm{c}} \mathrm{p}<0.05$ vs. chow $+\mathrm{E}-\mathrm{IH}$.

arteries from chow + E-IH rats compared to chow + air rats. Data for chow + air rats were repeated in this figure for ease of comparison. The antioxidant EUK-134 normalized vasodilation to the level of chow + air controls (fig. 6b).

Vasodilatory Responses in Arteries from HFD + Air Rats. Vasodilatory responses to ACh were affected in small arteries from HFD + air rats (fig. 6c) compared to arteries from chow + air rats (data repeated from fig. 6a). The antioxidant EUK-134 again normalized vasodilation to the level of chow + air controls.

Vasodilatory Responses in Arteries from HFD + E-IH Rats. ACh-induced dilation in arteries from HFD + E-IH rats was impaired to a similar degree as dilation in vessels from chow + E-IH rats (data from chow + E-IH repeated for comparison). There are no significant differences in the responsiveness of chow $+\mathrm{E}-\mathrm{IH}$ and HFD $+\mathrm{E}-\mathrm{IH}$ arteries (fig. 6d). Similar to results in the arteries from rats treated with just E-IH (chow + E-IH) or HFD (HFD + air), the antioxidant EUK-134 normalized vasodilation in these arteries.

\section{Discussion}

The major findings of this study were: (1) rats exposed to E-IH gain less weight and adiposity than their air-exposed counterparts; (2) combined high-fat feeding and $\mathrm{E}-\mathrm{IH}$ induces fasting hyperglycemia; (3) both E-IH and HFD increase oxidative stress; however, the effects are not additive; (4) increased ROS impair vasodilation in arteries

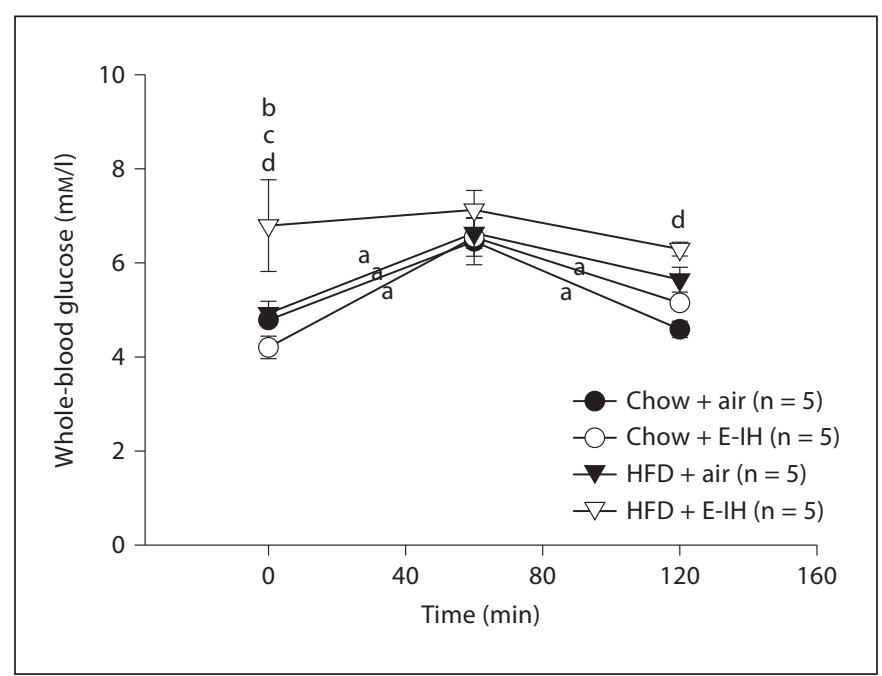

Fig. 3. OGTT: whole-blood glucose levels following an oral glucose dose $(1 \mathrm{~g} / \mathrm{kg})$ at time zero. HFD + E-IH rats had significantly elevated glucose levels prior to the start of the OGTT. Means \pm SEM for 5 animals per group. ${ }^{a} \mathrm{p}<0.05$ between time points; ${ }^{\mathrm{b}} \mathrm{p}<0.05$ HFD + E-IH vs. HFD + air; ${ }^{\mathrm{c}} \mathrm{p}<0.05$ HFD + E-IH vs. chow + E-IH; ${ }^{\mathrm{d}} \mathrm{p}<0.05$ HFD + E-IH vs. chow + air.

from both E-IH and HFD rats leading to elevated blood pressure; (5) ROS appear to contribute to vasodilation in arteries from control rats; (6) HFD does not exacerbate the impaired vasodilation or vascular levels of ROS caused by E-IH alone, and (7) although HFD + E-IH rats had increased oxidative stress compared to control rats, the levels were diminished in comparison to HFD + air alone. 


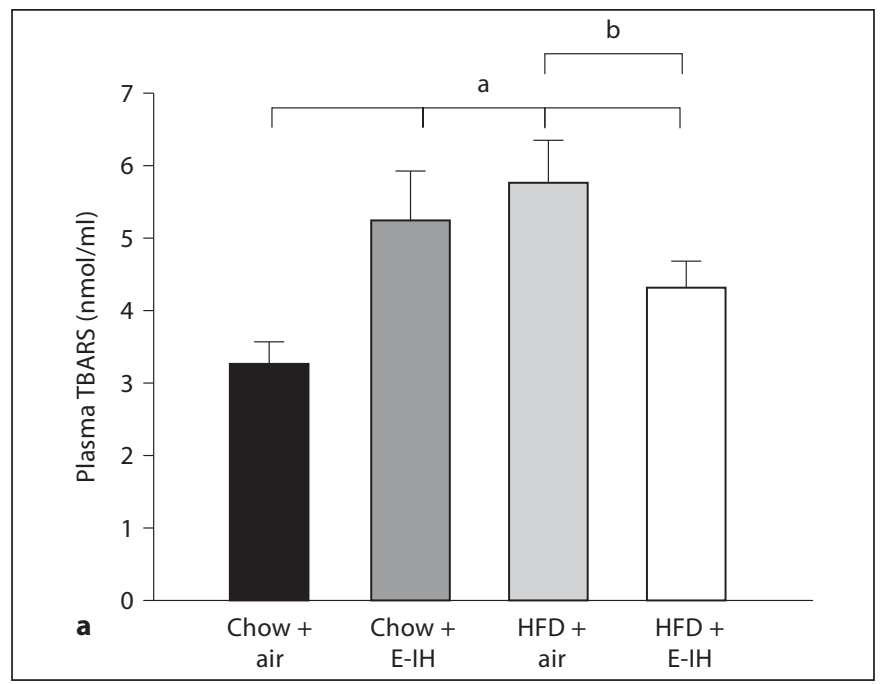

Fig. 4. Oxidative stress following air or E-IH exposure in chow and HFD rats. TBARS data demonstrate an increase in oxidative stress in animals exposed to E-IH with or without HFD ( $\mathrm{n}=14$ 16). DCF data demonstrate increased ROS in E-IH and HFD rats $(\mathrm{n}=7-10)$, suggesting an increase in $\mathrm{ONOO}^{-}$production as a result of superoxide scavenging of NO. The lack of increase in DCF

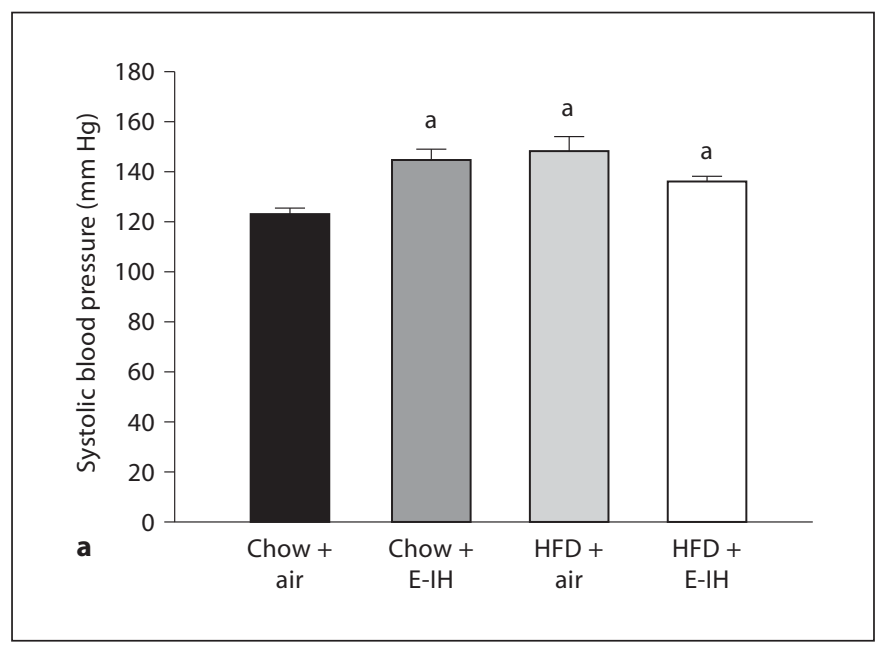

Fig. 5. Data are means \pm SEM. a Systolic blood pressure measurements following air or E-IH treatment in chow and HFD rats. Blood pressure was measured by tail cuff plethysmography. ${ }^{a} \mathrm{p} \leq$

These data suggest both elevated adiposity and simulated sleep apnea impair vascular function in an ROS-dependent manner; however, elevated adiposity does not exacerbate the vascular dysfunction in E-IH-exposed rats.

Rats exposed to simulated sleep apnea through E-IH have previously been shown to gain less weight than air-

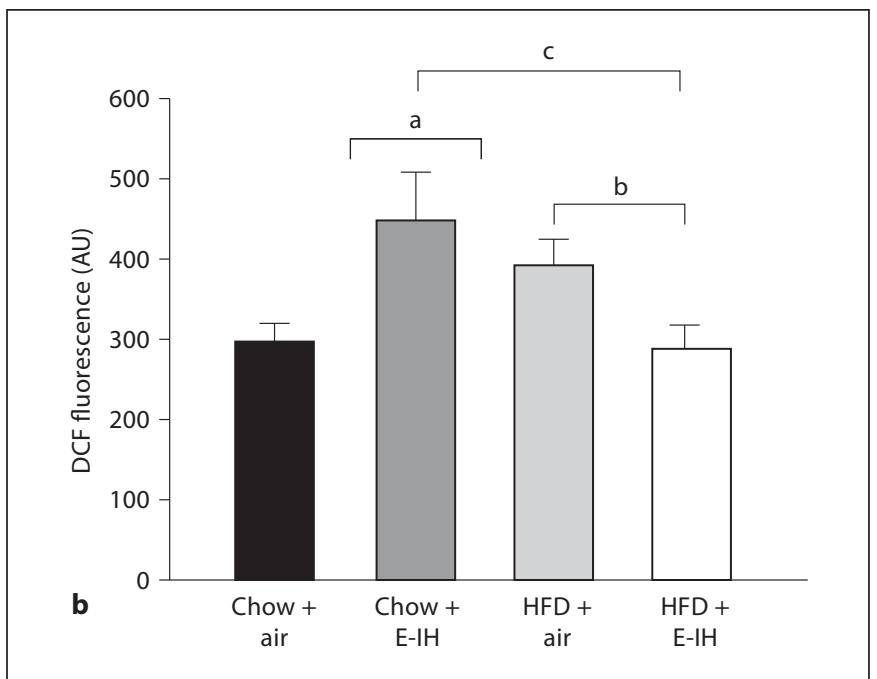

fluorescence in vessels from HFD + E-IH rats does not preclude the possibility of increased ROS in this group as levels of other ROS may be elevated as suggested by the TBARS data and ACh concentration response curves for this group. Means \pm SEM. ${ }^{\mathrm{a}} \mathrm{p}<0.05$ vs. chow + air; ${ }^{\mathrm{b}} \mathrm{p}<0.05$ vs. HFD + air; ${ }^{\mathrm{c}} \mathrm{p}<0.05$ vs. chow + E-IH.

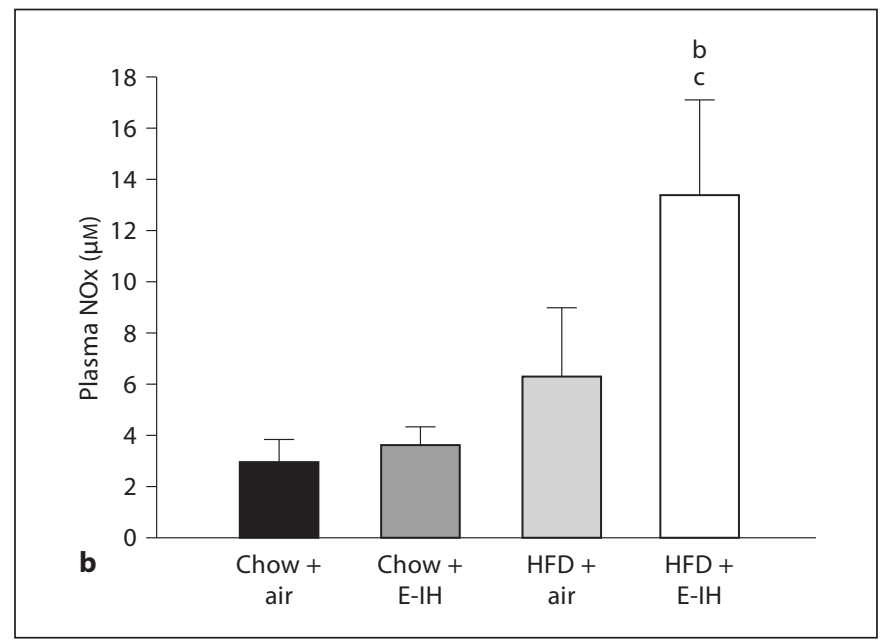

0.05 vs. chow + air, $\mathrm{n}=7-19 . \mathbf{b}$ Plasma nitrates and nitrites (NOx) levels in rats from all four groups. ${ }^{b} \mathrm{p}<0.05$ vs. HFD + air; ${ }^{c} \mathrm{p}<$ 0.05 vs. chow $+\mathrm{E}-\mathrm{IH}, \mathrm{n}=4-6$. 

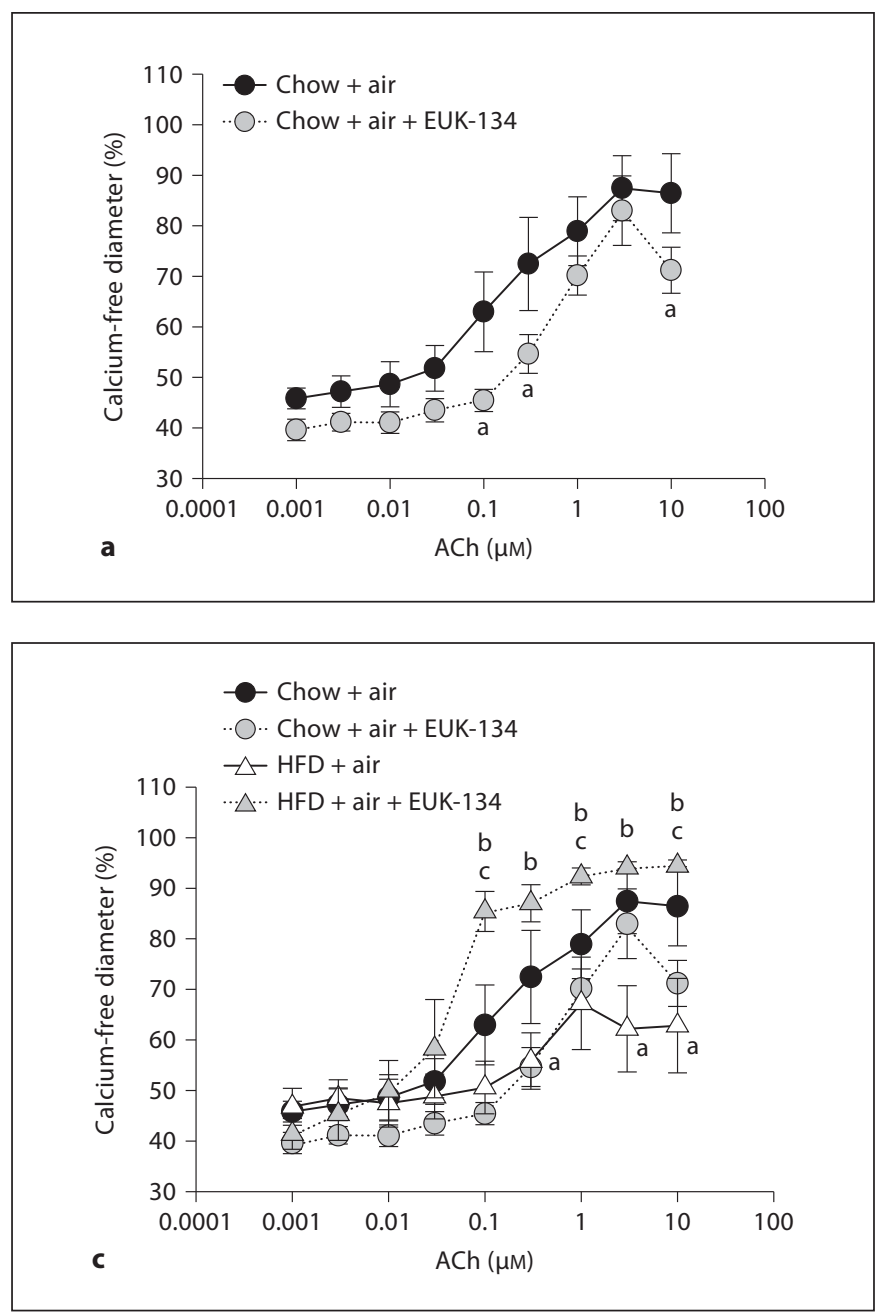

Fig. 6. Vasodilatory responses to the endothelium-dependent vasodilator ACh in mesenteric arteries from chow and HFD rats exposed to air or E-IH. a Vasodilatory responses in arteries from chow + air rats. b Vasodilation in vessels from chow + air rats compared to chow + E-IH rats. c Vasodilatory responses in HFD + air rats compared to similar treatments in arteries from chow + air rats. d Comparison of vasodilatory responses in vessels from

of diet, suggesting E-IH powerfully suppresses weight gain in rats (fig. 1). Sleep deprivation may play a role in decreased weight gain in rats exposed to E-IH since these animals tend to rouse intermittently during the hypoxic episodes [45].

It is possible that hypoxia-induced sleep deprivation decreases food intake although this was not assessed in the present study due to housing constraints in the hypoxic exposure system. Plasma levels of the adipose-derived hormone leptin were found to be elevated in HFD + air and HFD + E-IH rats above those of chow + air and
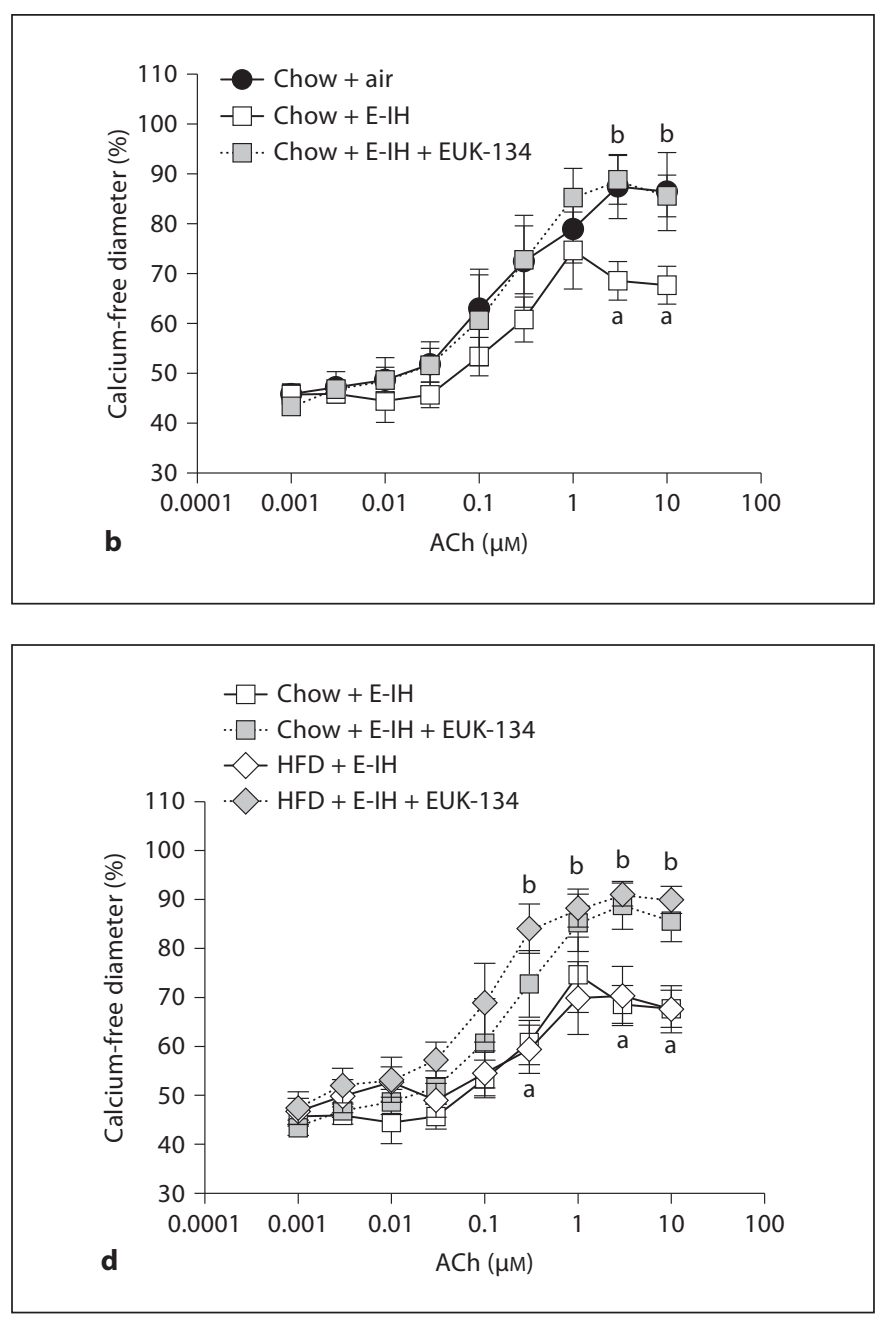

chow + E-IH and HFD + E-IH rats. There are no significant differences between HFD + E-IH and chow + E-IH at any dose. Data for chow + air and chow $+\mathrm{E}-\mathrm{IH}$ are repeated in the HFD figures to allow for visual comparisons between groups. Means \pm SEM. $\mathrm{n}=5$ per group. ${ }^{\mathrm{a}} \mathrm{p}<0.05$ vs. chow + air; ${ }^{\mathrm{b}} \mathrm{p}<0.05$ vs. respective control; ${ }^{\mathrm{c}} \mathrm{p}<0.05$ vs. chow + air + EUK-134.

chow + E-IH rats (fig. 2b). The diminished weight gain observed in the chow + E-IH rats is not associated with a rise in the levels of this satiety hormone (fig. 1a, 2b). Likewise, levels of leptin were not different in HFD + air and HFD + E-IH rats suggesting that leptin levels are more indicative of adiposity as opposed to contributing to the diminished weight gain through decreased appetite in these animals (fig. 2b).

Plasma corticosterone, the major glucocorticoid hormone released in rodents [37], was measured as a marker of stress but levels were not different between the chow + 
air and chow + E-IH groups (fig. 2a). Combined treatment of rats with HFD and E-IH resulted in greater plasma corticosterone levels than both the chow + air and chow + E-IH groups, but not significantly different from HFD + air (fig. 2a). These data demonstrate that while increased dietary fat intake leads to mild, but insignificant, increases in stress hormone levels, conditions that mimic sleep apnea significantly exacerbate stress in these animals.

Rats fed HFD and exposed to E-IH developed fasting (time 0) hyperglycemia compared to all other groups (fig. 3). These findings suggest that HFD and E-IH interact to further impair glucose handling compared to HFD + air or chow + E-IH alone. These data closely resemble those of humans with OSA who develop increased fasting plasma glucose levels compared to obese controls [35] as well as insulin resistance [19]. It is also established that increased corticosteroids can elicit insulin resistance [46, 47] and this may be a factor contributing to the fasting hyperglycemia observed in HFD + E-IH rats.

ACh-mediated vasodilation was significantly impaired in all three treatment groups (chow + E-IH, HFD + air, and HFD + E-IH; fig. 6) and correlated with elevated blood pressure (fig. 5a). One mechanism by which ACh elicits vasodilation is through NO. However, plasma NOx levels of chow + E-IH and HFD + air rats were not significantly different from chow + air rats (fig. 5b). Moreover, plasma NOx was markedly elevated in the HFD + E-IH rats (fig. 5b). However, this latter measure is reflective of basally produced $\mathrm{NO}$ in vivo and may not relate to ACh-induced activation of NOS. Furthermore, the NOx assay does not distinguish between bioavailable $\mathrm{NO}$ and NO that has been scavenged by ROS.

Vascular ROS appear to contribute to ACh-mediated vasodilation in arteries from chow + air rats since scavenging ROS with EUK-134 modestly attenuated dilation (fig. 6a). Although EUK-134 is a general ROS scavenger, $\mathrm{H}_{2} \mathrm{O}_{2}$ is most likely involved in this vasodilatory response in controls. Indeed, production of $\mathrm{H}_{2} \mathrm{O}_{2}$ is elevated in endothelial cells following ACh administration [48] and may elicit concentration-dependent vasodilation in mesenteric arteries of mice [48] although other studies observed vasoconstriction [10]. The ROS scavenger EUK134 used in the present study possesses enhanced catalase-like properties and thus is reportedly an effective scavenger of both $\mathrm{O}_{2}^{-}$and $\mathrm{H}_{2} \mathrm{O}_{2}$ [43].

In contrast to the results in arteries from chow-fed control rats, EUK-134 consistently improved vasodilatory responses to $\mathrm{ACh}$ in arteries from rats exposed to E-IH, HFD or their combination. This observation correlates with elevated TBARS and DCF fluorescence in ar- teries from these groups (fig. 4). Therefore, the attenuated vasodilation in vessels from these rats likely results from $\mathrm{O}_{2}^{-}$scavenging of $\mathrm{NO}$, producing $\mathrm{ONOO}^{-}$, since incubation with EUK-134 normalized vasodilation (fig. 6b) and there were either no differences in NOx (chow +E-IH and HFD + air) or levels were significantly elevated (HFD + E-IH; fig. 5b). Thus, ROS may contribute both to normal vasodilation $\left(\mathrm{H}_{2} \mathrm{O}_{2}\right)$ and to impaired vasodilation $\left(\mathrm{O}_{2}^{\overline{-}}\right)$ in these different experimental settings.

Obesity alone results in oxidative stress-mediated attenuation of endothelium-dependent vasodilation [28]. Similarly, patients with newly diagnosed OSA have reduced forearm blood flow responses to the endotheliumdependent vasodilator ACh compared to obese control patients [24]. In the present study, HFD rats and chow + E-IH rats had significantly increased ROS shown by elevated plasma TBARS and increased vascular DCF fluorescence (fig. 4). These results are similar to observations of elevated TBARS in patients with OSA compared to control subjects [49]. However, combining HFD and E-IH actually diminished DCF fluorescence and TBARS, although the TBARS values remained higher than those in chow + air controls (fig. 4).

Another effect of E-IH in the HFD rats was a decrease in body weight. Body weight was similar between chowand HFD-fed rats exposed to E-IH (fig. 1a); however, the HFD + E-IH rats maintained higher adiposity compared to chow + E-IH and chow + air rats (fig. 1b). The diminished adiposity in HFD + E-IH compared to HFD + air (fig. 1b) may have negated some of the effects of the HFD, especially if the effects of HFD alone are related to the degree of adiposity and not to the diet per se.

In conclusion, high-fat feeding and simulated sleep apnea both induce oxidative stress, elevated blood pressure, and impaired vasodilation but their effects do not appear to be additive. The lack of an additive effect of the two stimuli may relate to the effect of E-IH to reduce adiposity in HFD-fed animals.

\section{Acknowledgments}

This project was supported in part by the Dedicated Health Research Funds of the University of New Mexico School of Medicine (K.L.S. and B.R.W.) and HL-82799 (N.L.K.). The authors would like to thank Minerva Murphy for technical assistance and Christie Wilcox for assistance with OGTT. 


\section{References}

1 Baguet JP, Narkiewicz K, Mallion JM: Update on hypertension management: obstructive sleep apnea and hypertension. J Hypertens 2006;24:205-208.

$\checkmark 2$ Chen JK, Chow SE: Antioxidants and myocardial ischemia: reperfusion injuries. Chang Gung Med J 2005;28:369-377.

- 3 Kevin LG, Novalija E, Stowe DF: Reactive oxygen species as mediators of cardiac injury and protection: the relevance of anesthesia practice. Anesth Analg 2005;101:1275-1287.

-4 Madamanchi NR, Vendrov A, Runge MS: Oxidative stress and vascular disease. Arterioscler Thromb Vasc Biol 2005;25:29-38.

5 Sweazea KL, Walker BR: Antioxidant and vasodilatory effects of heme oxygenase on mesenteric vasoreactivity following chronic hypoxia. Microcirculation 2009;16:131-141.

-6 Taniyama Y, Griendling KK: Reactive oxygen species in the vasculature: molecular and cellular mechanisms. Hypertension 2003;42: 1075-1081.

7 Förstermann U: Janus-faced role of endothelial NO synthase in vascular disease: uncoupling of oxygen reduction from $\mathrm{NO}$ synthesis and its pharmacological reversal. Biol Chem 2006;387:1521-1533.

8 Kalinowski L, Malinski T: Endothelial NADH/NADPH-dependent enzymatic sources of superoxide production: relationship to endothelial dysfunction. Acta Biochim Pol 2004;51:459-469.

-9 Ray R, Shah AM: NAPDH oxidase and endothelial cell function. Clin Sci 2005; 109:217226.

10 Ardanaz N, Beierwaltes WH, Pagano PJ: Comparison of $\mathrm{H}_{2} \mathrm{O}_{2}$-induced vasoconstriction in the abdominal aorta and mesenteric artery of the mouse. Vasc Pharmacol 2007; 47:288-294.

11 Gil-Longo J, González-Vázquez C: Characterization of four different effects elicited by $\mathrm{H}_{2} \mathrm{O}_{2}$ in rat aorta. Vascul Pharmacol 2005; 43:128-138.

12 Mian KB, Martin W: Hydrogen peroxide-induced impairment of reactivity in rat isolated aorta: potentiation by 3-amino-1,2,4-triazole. Br J Pharmacol 1997;121:813-819.

13 Yamauchi M, Nakano H, Maekawa J, Okamoto Y, Ohnishi Y, Suzuki T, Kimura H: Oxidative stress in obstructive sleep apnea. Chest 2005;127:1674-1679.

14 Tassone F, Lanfranco F, Gianotti L, Pivetti S, Navone F, Rossetto R, Grottoli S, Gai V, Ghigo E, Maccario M: Obstructive sleep apnoea syndrome impairs insulin sensitivity independently of anthropometric variables. Clin Endocrinol 2003;59:374-379.

15 Chung S, Yoon I, Lee CH, Kim J: Effects of age on the clinical features of men with obstructive sleep apnea syndrome. Respiration 2009;78:23-29.
16 Palla A, Digiorgio M, Carpenè N, Rossi G, D’Amico I, Santini F, Pinchera A: Sleep apnea in morbidly obese patients: prevalence and clinical predictivity. Respiration 2009; 78:134-140.

17 Riha RL: Genetic aspects of the obstructive sleep apnoea/hypopnoea syndrome - is there a common link with obesity? Respiration 2009; 78:5-17.

18 Makino S, Handa H, Suzukawa K, Fujiwara M, Nakamura M, Muraoka S, Takasago I, Tanaka Y, Hashimoto K, Sugimoto T: Obstructive sleep apnoea syndrome, plasma adiponectin levels, and insulin resistance. Clin Endocrinol 2006;64:12-19.

19 Meslier N, Gagnadoux F, Giraud P, Person C, Ouksel H, Urban T, Racineux JL: Impaired glucose-insulin metabolism in males with obstructive sleep apnoea syndrome. Eur Respir J 2003;22:156-160.

20 Punjabi NM, Shahar E, Redline S, Gottlieb DJ, Givelber R, Resnick HE: Sleep-disordered breathing, glucose intolerance, and insulin resistance: the Sleep Heart Health Study. Am J Epidemiol 2004;160:521-530.

-21 Polotsky VY, Li J, Punjabi NM, Rubin AE, Smith PL, Schwartz AR, O’Donnell CP: Intermittent hypoxia increases insulin resistance in genetically obese mice. J Physiol 2003;552:253-264.

-22 Savransky V, Nanayakkara A, Li J, Bevans S, Smith PL, Rodriguez A, Polotsky VY: Chronic intermittent hypoxia induces atherosclerosis. Am J Respir Crit Care Med 2007;175: 1219-1221.

23 Lavie L, Polotsky V: Cardiovascular aspects in obstructive sleep apnea syndrome: molecular issues, hypoxia and cytokine profiles. Respiration 2009;78:361-370.

24 Kato M, Roberts-Thomson P, Phillips BG, Haynes WG, Winnicki M, Accurso V, Somers VK: Impairment of endothelium-dependent vasodilation of resistance vessels in patients with obstructive sleep apnea. Circulation 2000;102:2607-2610.

25 Chung S, Yoon I, Lee CH, Kim J: The association of nocturnal hypoxemia with arterial stiffness and endothelial dysfunction in male patients with obstructive sleep apnea syndrome. Respiration 2010;79:363-369.

26 Ip MSM, Tse H, Lam B, Tsang KWT, Lam W: Endothelial function in obstructive sleep apnea and response to treatment. Am J Respir Crit Care Med 2004;169:348-353.

27 Grebe M, Eisele HJ, Weissmann N, Schaefer C, Tillmanns H, Seeger W, Schultz R: Antioxidant vitamin $\mathrm{C}$ improves endothelial function in obstructive sleep apnea. Am J Respir Crit Care Med 2006;173:897-901.

28 Romanko OP, Stepp DW: Reduced constrictor reactivity balances impaired vasodilation in the mesenteric circulation of the obese Zucker rat. Am J Physiol Heart Circ Physiol 2005;289:H2097-H2101.
29 Song GY, Gao Y, Di YW, Pan LL, Zhou Y, Ye JM: High-fat feeding reduces endotheliumdependent vasodilation in rats: differential mechanisms for saturated and unsaturated fatty acids? Clin Exp Pharmacol Physiol 2006;33:708-713.

30 Keaney JF, Xu A, Cunningham D, Jackson T, Frei B, Vita JA: Dietary probucol preserves endothelial function in cholesterol-fed rabbits by limiting vascular oxidative stress and superoxide generation. J Clin Invest 1995;95: 2520-2529.

31 Sweazea KL, Lekic M, Walker BR: Comparison of mechanisms involved in impaired vascular reactivity between high sucrose and high fat diets in rats. Nutr Metab 2010;7:48.

32 Allahdadi KJ, Walker BR, Kanagy NL: Augmented endothelin vasoconstriction in intermittent hypoxia-induced hypertension. Hypertension 2005;45:705-709.

33 Kanagy NL, Walker BR, Nelin LD: Role of endothelin in intermittent hypoxia-induced hypertension. Hypertension 2001;37:511515.

-34 Shamsuzzman AS, Gersh BJ, Somers VK: Obstructive sleep apnea: implications for cardiac and vascular disease. JAMA 2003; 290:1906-1914.

- 35 Vgontzas AN, Papanicolaou DA, Bixler EO, Hopper K, Lotsikas A, Lin H, Kales A, Chrousos GP: Sleep apnea and daytime sleepiness and fatigue: relation to visceral obesity, insulin resistance, and hypercytokinemia. J Clin Endocrinol Metab 2000;85: 1151-1158.

36 Snow JB, Kitzis V, Norton CE, Torres SN, Johnson KD, Kanagy NL, Walker BR, Resta TC: Differential effects of chronic hypoxia and intermittent hypocapnic and eucapnic hypoxia on pulmonary vasoreactivity. J Appl Physiol 2008;104:110-118.

37 Thomas P, Wofford HW: High-performance liquid chromatography of corticosteroids in vertebrate plasma: assay of cortisol in mullet and corticosterone in the rat. Comp Biochem Physiol B 1984;78:473-479.

38 Christensen KL, Mulvany MJ: Mesenteric arcade arteries contribute substantially to vascular resistance in conscious rats. J Vasc Res 1993;30:73-79.

39 Crow JP: Dichlorodihydrofluorescein and dihydrorhodamine 123 are sensitive indicators of peroxynitrite in vitro: implications for intracellular measurement of reactive nitrogen and oxygen species. Nitric Oxide 1997;1:145-157.

40 Keller A, Mohamed A, Dröse S, Brandt U, Fleming I, Brandes RP: Analysis of dichlorodihydrofluorescein and dihydrocalcein as probes for the detection of intracellular reactive oxygen species. Free Radic Res 2004;38: 1257-1267. 
-41 Myrhe O, Andersen JM, Aarnes H, Fonnum F: Evaluation of the probes $2^{\prime}-7^{\prime}$-dichlorofluorescein diacetate, luminol, and lucigenin as indicators of reactive oxygen species formation. Biochem Pharmacol 2003;65:15751582.

42 Jernigan NL, Resta TC, Walker BR: Contribution of oxygen radicals to altered $\mathrm{NO}$-dependent pulmonary vasodilation in acute and chronic hypoxia. Am J Physiol Lung Cell Mol Physiol 2004;286:L947-L955.
43 Baker K, Marcus CB, Huffman K, Kruk H, Malfroy B, Doctrow SR: Synthetic combined superoxide dismutase/catalase mimetics are protective as a delayed treatment in a rat stroke model: a key role for reactive oxygen species in ischemic brain injury. J Pharmaco Exp Ther 1998;284:215-221.

44 Day B: Catalytic antioxidants: a radical approach to new therapeutics. Drug Discov Today 2004;9:557-566.

-45 Spiegel K, Leproult R, Van Cauter E: Impact of sleep debt on metabolic and endocrine function. Lancet 1999;354:1435-1439.

-46 Kajita K, Ishizuka T, Miura A, Kanoh Y, Ishizawa M, Kimura M, Muto N, Yasuda K: Glucocorticoid-induced insulin resistance associates with activation of protein kinase C isoforms. Cell Signal 2001;13:169-175.
7 Leboeuf B, Renold AE, Cahill GF Jr: Studies on rat adipose tissue in vitro. IX. Further effects of cortisol on glucose metabolism. J Biol Chem 1962;237:988-991.

48 Matoba T, Shimokawa H, Nakashima M, Hirakawa Y, Mukai Y, Hirano K, Kanaide $\mathrm{H}$, Takeshita A: Hydrogen peroxide is an endothelium-derived hyperpolarizing factor in mice. J Clin Invest 2000;106:1521-1530.

49 Lavie L, Vishnevsky A, Lavie P: Evidence for lipid peroxidation in obstructive sleep apnea. Sleep 2004;27:123-128. 